\title{
A Novel Subspace Based DOA Estimation Method for Vehicular
} Application

\author{
JuQi SHI ${ }^{1, a}$, XiaoYou YU ${ }^{1, b^{*}}$, Heng YE ${ }^{1}$, Yan $\mathrm{ZHOU}^{1}$, Nengda $\mathrm{Cl}^{1}$, \\ Pengshuai SUN ${ }^{1}$, Zhinian LUO ${ }^{1}$ \\ ${ }^{1}$ College of Information Science and Engineering, Hunan University, Changsha 410012, China \\ aemail: 444599327@qq.com, bemail: yu.xiaoyou@163.com
}

Keywords: Vehicular Communication System; DOA Estimate Method; Subspace

\begin{abstract}
In vehicular communication system, there is only a small number of samples for real time location system, so the sample covariance matrix will be a large degree of deviation from the signal covariance matrix. To solve this problem, a new method of DOA estimation is proposed in this paper, which improves the performance of DOA Estimation by modifying the sample covariance matrix. Simulation results illustrate the improvement achieved by the new method and demonstrate it is suitable for vehicular application.
\end{abstract}

\section{Introduction}

In recent years, vehicular communication [1,2] has become a hot research topic in the intelligent transportation system[3,4]. By MIMO-OFDM signal, Vehicular communication system perceive the surrounding environment changes and traffic conditions, and the MIMO antenna to determine the vehicle azimuth angle, finally, realizing the multiple objective location [5,6].

Because the integration system is in a high speed moving communication environment, the signal received by the user changes very quickly. DOA estimation methods, such as MUSIC algorithm [7], root MUSIC algorithm [8], Propagation operator algorithm [9] are based on the number of samples according to the covariance matrix. Although these algorithms have good performance of the super-resolution, but they need feature decomposition and spectral peak searching, the computational is very large. In small samples, these algorithm performance sharply decline. Therefore, these algorithm can't meet the demand of vehicular application. In the case of small samples, the sample data covariance matrix may deviate significantly from the actual signal covariance matrix. Literature [10] shows that the performance decline is related to the subspace leakage caused by the noise subspace, and the subspace leakage problem is introduced in detail.

In order to meet the requirement of car networking[11], this paper proposed a new method that needn't spectral peak searching, which greatly reduces the amount of computation. Through update the covariance matrix of the sample data to solve the performance caused by the small number of samples decreased. Simulation results show that the new method is superior, and it lays a solid foundation for the real-time positioning of the vehicle networking.

\section{System Model}

The vehicle carrying the integrated system transmits MIMO-OFDM signal to the vehicle in the road, and the user receives the signal transmitted by the target vehicle. User can detect the orientation of the target vehicle according to the received signal through the appropriate processing algorithm. Due to the different azimuth angles of the target vehicle and the user, the integrated system of vehicle communication uses MIMO antenna processing technique to estimate the DOA of the target signal. Under the 802.11 p standard, the whole process can be regarded as the point to point target detection. This paper assumes that the target from different directions.

For studing convenient, based on the hypothesis that the vehicular communication system of MIMO antenna array is uniform linear array, which is shown in Fig 1. The number of antennas at the transmitting end and the receiving end is $M_{T}$ and $M_{R}$. Fig.1 show signal receiving model in 
vehicle environment, $\theta_{i}$ indicates the angle between the ith target signal and the array normal, the antenna array element spacing is $\mathrm{d}(d \leq \lambda / 2) . \lambda$ is the plane wave length that arrived antenna array.

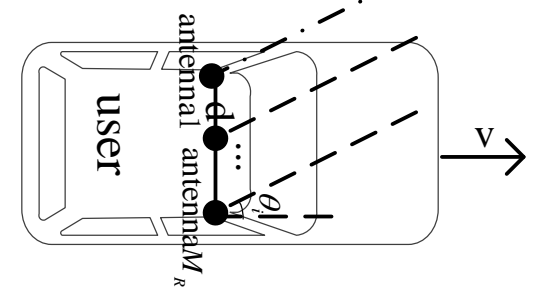

Fig.1. signal receiving model in vehicle environment

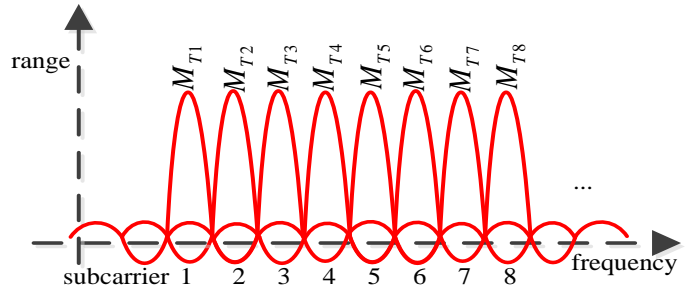

Fig. 2 antenna allocation scheme

Assuming the number of transmit antennas $M_{T}=4$. Figure 2 shows the distribution scheme for the transmitter antenna.

The signal from the antenna $p\left(p=0,1, \cdots, M_{T}\right)$ can be expressed as:

$$
s_{p}(t)=\sum_{\mu=1}^{N_{s_{m}}} \sum_{n=1}^{N_{c} / M_{T}} d_{T}\left(\mu N_{c} / M_{T}+n\right) \cdot \exp \left(j 2 \pi\left(p+n M_{T}\right) \Delta f t\right) \operatorname{rect}\left(\frac{t-\mu T}{T}\right)
$$

Where $N_{\text {sym }}$ is a number of OFDM symbol, $N_{c} / M_{T}$ is the number of subcarriers assigned to one antenna ( $\mathrm{N}_{\mathrm{c}}$ is the total number of subcarriers used for all antennas), $d_{T}$ is modulated data, $\Delta f=1 / T$ is the spacing between adjacent subcarriers. Therefore, $s_{i}(t)=\sum_{p=1}^{M_{T}} s_{p}(t)$.

Due to the slight difference in the transmit-receive antenna array positions, the received signal at the $q \operatorname{th}\left(q=0,1, \cdots, M_{R}\right)$ receive antenna can be expressed as:

$$
x_{q}(t)=\sum_{p=1}^{M_{T}} \sum_{\mu=1}^{N_{s m}} \sum_{n=1}^{N_{c} M_{T}} d_{R}\left(\mu N_{c} / M_{T}+n\right) \cdot \exp \left(j 2 \pi\left(p+n M_{T}\right) \Delta f t\right) \operatorname{rect}\left(\frac{t-\mu T}{T}\right)
$$

Where, $d_{R}=\beta \cdot d_{T} \cdot \exp (-j 2 \pi \Delta f \tau), \beta$ denotes the complex amplitude attenuation during propagation to the object, $\tau=d \sin \left(\theta_{i}\right) / \lambda$.

Therefore, the received signal within the instantaneous time can be expressed as:

$$
\mathbf{x}(\mathrm{t})=\sum_{q=1}^{M_{R}} x_{q}(t)+\boldsymbol{n}(t)=\beta \sum_{\mu=1}^{N_{s y m}} \sum_{n=1}^{N_{c} / M_{T}} \mathbf{a}\left(\theta_{i}\right) s_{i}(t)+\mathbf{n}(t)
$$

$\mathbf{a}\left(\theta_{i}\right)=\left[1, \exp \left(-j 2 \pi d / \lambda \sin \left(\theta_{i}\right), \cdots, \exp \left(-j 2 \pi(M-1) d / \lambda \sin \left(\theta_{i}\right)\right)\right]^{T}\right.$ is the direction vector of $i$ signal, $\mathbf{n}(\mathrm{t})$ is noise vector.

\section{New DOA Estimate Method}

At the receiving end, the noise source can be seen as independent of time $t$, Number of receiving samples is $N$, thus the estimated value of receiving samples data $\mathbf{x}(t)(1 \leq t \leq N)$ covariance matrix is given by:

$$
\begin{aligned}
\hat{\mathbf{R}} & \triangleq \frac{1}{N} \sum_{t=1}^{N}(\mathbf{A} \beta \mathbf{s}(t)+\mathbf{n}(t))(\mathbf{A} \beta \mathbf{s}(t)+\mathbf{n}(t))^{H} \\
& =\mathbf{A}\left\{\frac{\beta^{2}}{N} \sum_{t=1}^{N} \mathbf{s}(t) \mathbf{s}^{H}(t)\right\} \mathbf{A}^{H}+\frac{1}{N} \sum_{t=1}^{N} \mathbf{n}(t) \mathbf{n}^{H}(t)+\mathbf{A}\left\{\frac{\beta}{N} \sum_{t=1}^{N} \mathbf{s}(t) \mathbf{n}^{H}(t)\right\}+\left\{\frac{\beta}{N} \sum_{t=1}^{N} \mathbf{n}(t) \mathbf{s}^{H}(t)\right\} \mathbf{A}^{H}
\end{aligned}
$$

Where $\mathbf{A} \in \mathbb{C}^{M \times K}$ is direction matrix, $\mathbf{A}=\left[\mathbf{a}\left(\theta_{1}\right), \mathbf{a}\left(\theta_{2}\right), \ldots, \mathbf{a}\left(\theta_{K}\right)\right], \quad \mathbf{I}_{M} \in \mathbb{C}^{M \times M}, \quad \mathbf{S}=\mathrm{E}\left\{\mathbf{s}(\mathrm{t}) \mathbf{s}^{\mathrm{H}}(\mathrm{t})\right\}$ is the source covariance matrix, $(\cdot)^{H}$ stand for the Hermitian transposition.

The first two terms of (4) is the signal and noise components. The last two terms are undesirable by-products. Under the study of system model, we consider the signal and noise components are uncorrelated to each other. As a result, for a large enough number of samples $\mathrm{N}$, the last two terms tend to zero. However, the number of available samples in vehicle environment can be limited in practical applications. In this case, the last two terms may have significant values. Therefore, we 
update the covariance matrix of the sample data.

The third term in (4) can be signed as $\mathbf{T}=\hat{\mathbf{P}}_{A} \hat{\mathbf{R}} \mathbf{P}_{A}^{\perp}, \quad \hat{\mathbf{P}}_{A}$ is an estimation for the projection matrix of the signal subspace, given by $\hat{\mathbf{P}}_{A}=\hat{\mathbf{A}}\left(\hat{\mathbf{A}}^{\mathrm{H}} \hat{\mathbf{A}}\right) \hat{\mathbf{A}}^{\mathrm{H}}, \hat{\mathbf{A}}$ is estimated direction matrix, $\mathbf{P}_{A}^{\perp}$ is an estimation for the projection matrix of the noise subspace, The forth term is equal to the Hermitian of the third term $\mathbf{T}^{H}$, Finally, the modified data covariance matrix is obtained by deducting a scaled version of the estimated terms from the initial sample data covariance matrix as $\hat{\mathbf{R}}^{(2)}=\hat{\mathbf{R}}-\gamma\left(\mathbf{T}+\mathbf{T}^{H}\right), \lambda \in(0,1]$. Therefore, the differences between the observations and the estimates can be reduced.

Divided matrix $\hat{\mathbf{A}}$ into two submatrices $\mathbf{A}_{1}$ and $\mathbf{A}_{2}, \mathbf{A}_{1} \in \mathbb{C}^{M \times M}, \mathbf{A}_{2} \in \mathbb{C}^{(M-K) \times K}, \mathbf{A}_{1}$ have full rank. Hence $\mathbf{A}_{1}$ is invertible, there is a linear operator $\mathbf{J} \in \mathbb{C}^{K \times(M-K)}$ make $\mathbf{A}_{2}=\mathbf{J}^{H} \mathbf{A}_{1}$.

The received signal vector $\mathbf{A}$ is similar to $\hat{\mathbf{R}}$ that of a subspace, thus, in the absence of noise.

$$
\hat{\mathbf{R}}^{(2)}=\frac{\beta^{2}}{N} \hat{\mathbf{A}} \mathbf{S} \hat{\mathbf{A}}^{H}=\frac{\beta^{2}}{N}\left[\begin{array}{cc}
\mathbf{A}_{1} \mathbf{S} \mathbf{A}_{1}^{H} & \mathbf{A}_{1} \mathbf{S A}_{1}^{H} \mathbf{J} \\
\mathbf{J}^{H} \mathbf{A}_{1} \mathbf{S A}_{1}^{H} & \mathbf{J}^{H} \mathbf{A}_{1} \mathbf{S A}_{1}^{H} \mathbf{J}
\end{array}\right]
$$

Similarly, The matrix $\hat{\mathbf{R}}$ is divided into two submatrices $\mathbf{R}_{1}$ and $\mathbf{R}_{2}, \mathbf{R}_{1} \in \mathbb{C}^{K \times K}, \mathbf{R}_{2} \in \mathbb{C}^{(M-K) \times K}$, $\mathbf{J}=\left(\mathbf{A}_{1}^{H}\right)^{-1} \mathbf{A}_{2}=\left(\mathbf{R}_{1}^{H}\right)^{-1} \mathbf{R}_{2}$. Defining the matrix $\mathbf{G} \triangleq\left[\mathbf{J}^{T},-\mathbf{I}_{M-K}\right]^{T}, \hat{\mathbf{A}}=\left[\mathbf{A}_{1}^{T}, \mathbf{A}_{2}^{T}\right]^{T}$, so $\mathbf{G}^{H} \hat{\mathbf{A}}=\mathbf{0}_{(M-K) \times K}$, In the condition of small samples, using root MUSIC algorithm get the DOA estimate value at first step, then updating the sample covariance matrix to reduce the leakage of the signal subspace. Finally we can obtain the function by the formula $f(\theta)$, To minimize $f(\theta)$ we can obtain the estimated value :

$$
\hat{\theta}_{i}=\arg \min f(\theta) \triangleq \arg \boldsymbol{a}^{H}\left(\theta_{i}\right) \hat{\mathbf{G}} \hat{\mathbf{G}}^{H} \boldsymbol{a}\left(\theta_{i}\right)
$$

\section{Simulation Results and Performance Analysis}

Simulation experiments based on IEEE 802.11p standard under the vehicle networking. Table 1 shows the standard simulation parameters. In the simulation, the proposed method and root MUSIC algorithm applied to the system. There are three targets considered in the simulation. Simulation including DOA estimation, algorithms RMSE simulation compare under different samples.

Table 1 The simulation parameters

\begin{tabular}{ccccccc}
\hline $\mathrm{N}_{\mathrm{c}}$ & $\Delta \mathrm{f}(\mathrm{kHz})$ & $\mathrm{f}_{\mathrm{c}}(\mathrm{GHz})$ & $\mathrm{d}$ & $\mathrm{M}_{\mathrm{T}}$ & $\mathrm{M}_{\mathrm{R}}$ & $\theta\left(^{\circ}\right)$ \\
\hline 64 & 625 & 5.9 & $\lambda / 2$ & 4 & 4 & $0,30,60$ \\
\hline
\end{tabular}

Fig. 3 shows DOA estimation of the two methods. The estimation results of root MUSIC and the new method are-0.20328,29.8457, 60.9448 and-0.0036,30.0004,60.003, respectively. It can be seen that the new method can achieve the same super-resolution estimation as root MUSIC, even slightly better.
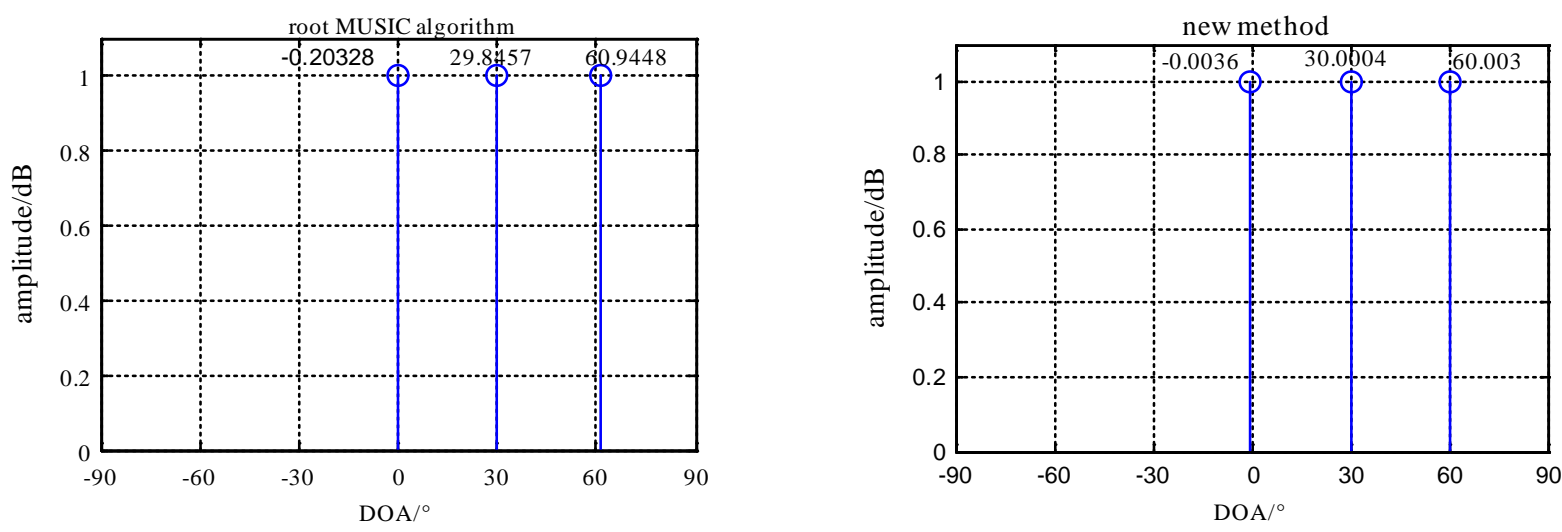

Fig. 3 DOA estimation results of the two algorithms

To describe the angular accuracy of the two algorithm, defining RMSE (root mean square error)is:

$$
\text { RMSE }=\sqrt{\sum_{i=1}^{n}\left(\hat{\theta}_{i}-\theta_{0}\right)^{2} / n}
$$

The number of Monte-Carlo experiments is 100 at each sample and result is shown in Fig. 4, 
which indicates that the performance of the new method is better than root MUSIC algorithm at small samples. It can be seen that the new method can obtained the angular close to the real value.

The new method can obtain better performance in small sample size, it is a better algorithm.

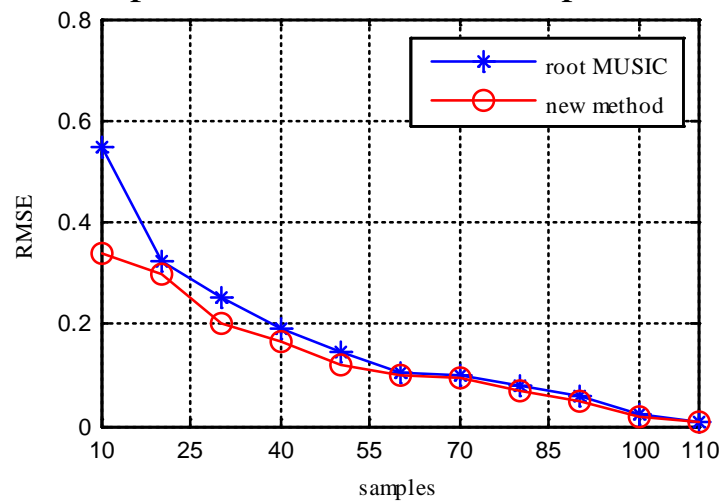

Fig 4. RMSE of the two algorithms

\section{Conclusion}

According to the requirements of vehicle networking, to improve the performance of DOA estimation algorithm in small samples, this paper presents a new method to update the covariance matrix of the two step, which has good performance and suitable for vehicular application. The new method can also be updated in three steps to achieve a higher angle estimation accuracy. Experiments show that the new method is of high accuracy and can improve the engineering realization of the integrated system in the vehicle networking application environment.

\section{Acknowledgement}

In this paper, the research was sponsored by the National Natural Science Foundation of China (Project No. 61371115).

\section{References}

[1] Si W, Si W. Integrating wireless technologies into intra-vehicular communication [J]. Dissertations \& Theses - Gradworks, 2016.

[2] Sharef B T, Alsaqour R A, Ismail M. Vehicular communication ad hoc routing protocols: A survey [J]. Journal of Network \& Computer Applications, 2014, 40(2):363-396.

[3] D. Kandar, V. Dhilip Kumar, Phida Chyne. OFDM Simulation in Combined WiMAX/DSRC Mechanism for Intelligent Transportation System [J]. 2016.

[4] Sturm C, Wiesbeck W. Waveform Design and Signal Processing Aspects for Fusion of Wireless Communications and Radar Sensing [J]. Proceedings of the IEEE, 2011, 99(7):1236-1259.

[5] Han G, Wan L, Shu L, et al. Two Novel DOA Estimation Approaches for Real-Time Assistant Calibration Systems in Future Vehicle Industrial [J]. IEEE Systems Journal, 2015:1-12.

[6] Spadafora W G, Paielli P M, Llewellyn D R, et al. Intelligent transportation system:, US 7689230 B2[P]. 2010.

[7] Vallet P, Mestre X, Loubaton P. Performance Analysis of an Improved MUSIC DOA Estimator [J]. Signal Processing IEEE Transactions on, 2015, 63(23):6407-6422.

[8] Wei Wang, Xianpeng Wang, Xin Li, et al. DOA estimation for monostatic MIMO radar based on unitary root-MUSIC[J]. International Journal of Electronics, 2013, 100(11):1499-1509.

[9] $\mathrm{Hu}$ W W, Wang C M, Zhang A J. Application of Weighted Projection PM Algorithm in Estimation of DOA[J]. Applied Mechanics \& Materials, 2013, 300-301(8):746-749. 
[10] Shaghaghi M, Vorobyov S. Subspace Leakage Analysis and Improved DOA Estimation with Small Sample Size [J]. Signal Processing IEEE Transactions on, 2015, 63:3251-3265.

[11]He W, Li Z, Xie G. Research and Development Trends of Car Networking [J]. Communications in Computer \& Information Science, 2010, 134:296-301. 